\title{
FLEXIBLE WIDE-BAND TWO-ARM SPIRAL ANTENNA FOR DIRECTIVITY OPTIMIZATION
}

\author{
Peng Liu, Siming Yang, Qiugu Wang, Mingda Yang, Jiming Song, and Liang Dong* \\ Iowa State University, Ames, Iowa, USA
}

\begin{abstract}
This paper reports a directivity-reconfigurable antenna with a wide frequency operation range in microwave regime. The device is formed by encasing liquid metal alloys into a highly stretchable elastomer membrane. The antenna is designed to have a two-arm Archimedean spiral structure and is able to optimize its directivity by inflating the elastomer to form a dome-shaped structure with different heights, pneumatically driven by microelectromechanical systems (MEMS) based microblowers. The antenna can operate in a wide frequency band from $6.9 \mathrm{GHz}$ to $13.8 \mathrm{GHz}$. The proposed tuning mechanism allows optimizing radiation patterns of the antenna by making radiation become more directive in the inflation direction, while the passing band of the device still remains wide.
\end{abstract}

\section{INTRODUCTION}

Flexible electronics have been intensively studied because they are able to be conveniently integrated on curved surfaces for a broad range of applications such as wearable devices, artificial skins, flexible displays, sustainable energy harvesters [1-3]. Recently, inclusion of liquid metal into stretchable elastomers has opened up many great opportunities to develop tunable and flexible electronic and electromagnetic devices in radio frequency range [4-6]. Generally, liquid metals and alloys can flow and reshape even under large mechanical deformation. Gallium-Indium eutectic (EGaIn) is considered as a promising substitution of mercury because of its liquid state in room temperature and low toxicity. Recently, we have recently developed a fabrication process capable of embedding EGaIn into a stretchable silicone-based elastomer (Ecoflex). This process has allowed us to develop stretchable and tunable microwave meta-atoms [6], and meta-skins for tunable frequency selective surface and microwave cloak applications [7], where an array of EGaIn split ring resonators are encased into Ecoflex.

Reconfigurable antennas have attracted increasing attention due to the explosive growth of wireless communications. Several liquid metal based tunable antennas have been reported based on different tuning mechanisms and designs, including a stretchable dipole antenna or unbalanced loop antenna [8], an electrically small antenna [9], and an electrochemical controlled capillary actuated monopole antenna [10]. All of the existing devices have focused on frequency tuning without paying much attention to optimization of directivity. It should be noted that directivity reconfigurability is highly desirable for applications, such as remote sensing and targeted power transfer, where signal and power strength in a specific direction needs to be improved.

Here, we report a flexible and inflatable two-arm spiral antenna for directivity optimization in microwave regime. The antenna is formed by encasing liquid metal into a highly stretchable elastomer. The antenna can be inflated from its original planar shape to a dome-like shape by blowing high-pressure air into an air cavity below the antenna using microelectromechanical systems (MEMS) based microblowers. The antenna is capable of changing its shape and allows reconfiguring the radiation pattern of the antenna along the main lobe direction or the inflation direction. When the antenna is inflated to the dome-like shape, the radiation pattern of the antenna becomes shaper, thus optimizing its directivity, while its reflection coefficient remains almost unchanged in a wide band range. In addition, the antenna provides good circular polarization.

\section{DESIGN AND FABRICATION}

Fig. 1(a) shows a schematic for a planar self-complementary, two-arm Archimedean spiral antenna made of liquid metal encased by a silicone elastomer (Ecoflex, Smooth-On, Macungie, PA). The operating frequency range of the antenna can be determined by the inner radius $a$ and the outer radius $b$. In order to operate in X-band regime, $a$ and $b$ of are set to be $3.5 \mathrm{~mm}$ and $18.5 \mathrm{~mm}$, respectively. Each spiral arm has 2.5 turns. The width of the arm is $w=1.5 \mathrm{~mm}$ and the gap between two arms is $g=1.5 \mathrm{~mm}$.
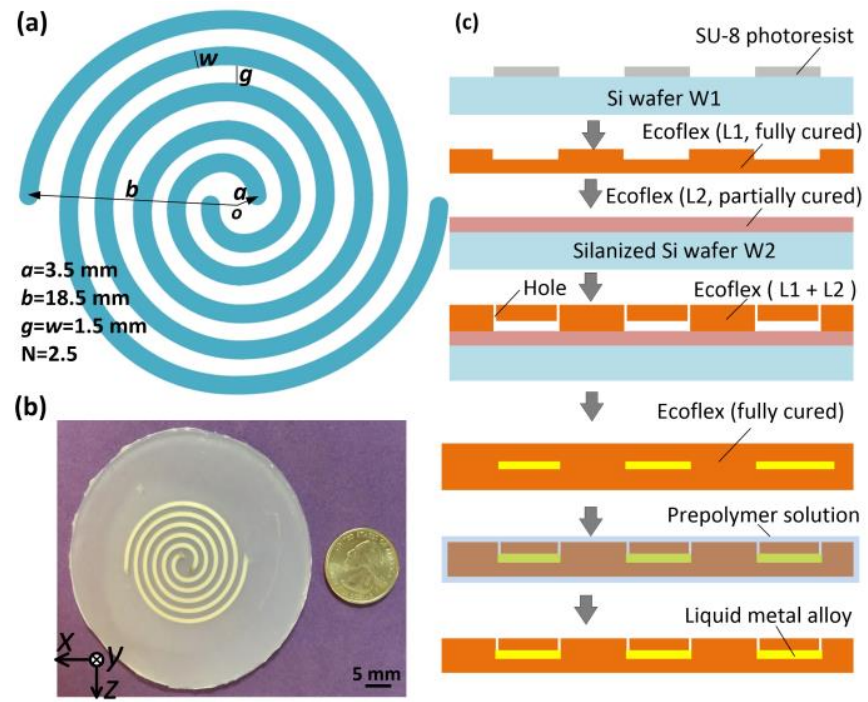

Figure 1: (a) Design parameters of the proposed two-arm spiral antenna. (b) Photo of the fabricated planar antenna before assembled to a pneumatic control unit. (c) Schematic of the fabrication process for the planar antenna.

Fig. 1(c) schematically shows the fabrication process of the antenna. First, a SU-8 photoresist (Microchem, Westborough, MA) is patterned to the designed spirals structure on a silicon wafer W1 with traditional photolithography. Subsequently, a $700 \mu \mathrm{m}$ thick silicone layer L1 is formed by casting a prepolymer solution of silicone Ecoflex on the SU-8 mold, and fully thermally cured at $60{ }^{\circ} \mathrm{C}$ on a hotplate for $30 \mathrm{~min}$. The fully cured L1 layer is then peeled from the SU-8 mold with the spiral channels inside it. An inlet and an outlet are mechanically punched at the two ends of each channel as the liquid metal injection vias. Due to the poor adhesion between fully cured Ecoflex, another $100 \mu \mathrm{m}$ thick Ecoflex layer L2 is spin coated and followed by a partial curing process on a silanized bare Si wafer W2 at $45^{\circ} \mathrm{C}$ for $40 \mathrm{sec}$. After that, the fully cured L1 and the partially cured L2 are immediately transferred to a hotplate at $90^{\circ} \mathrm{C}$ for 20 mins. This allows permanently bonding these two layers. Thus, the spiral channels are formed inside the elastomer with inlet and outlet. After EGaIn is manually injected into the channels with a metal needle (\#20 Gauge) amounted on a syringe $(10 \mathrm{ml}$; Becton-Dickinson, Franklin Lakes, NJ), residues of liquid 
metal around the inlets and outlets are cleaned by a cotton swab dipped with a hydrochloric acid solution $(50 \%, \mathrm{v} / \mathrm{v})$. Lastly, the device is immersed into a thin prepolymer solution of Ecoflex and then is fully cured at $80{ }^{\circ} \mathrm{C}$ for $30 \mathrm{~min}$ to seal the inlets and outlets. The total thickness of the elastomer is approximately $1 \mathrm{~mm}$. The liquid metal is located in nearly half the thickness of the elastomer (Fig. 1(b),(c)).

(a)

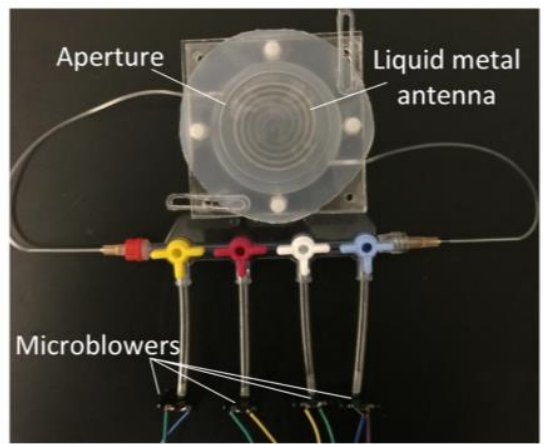

(b)

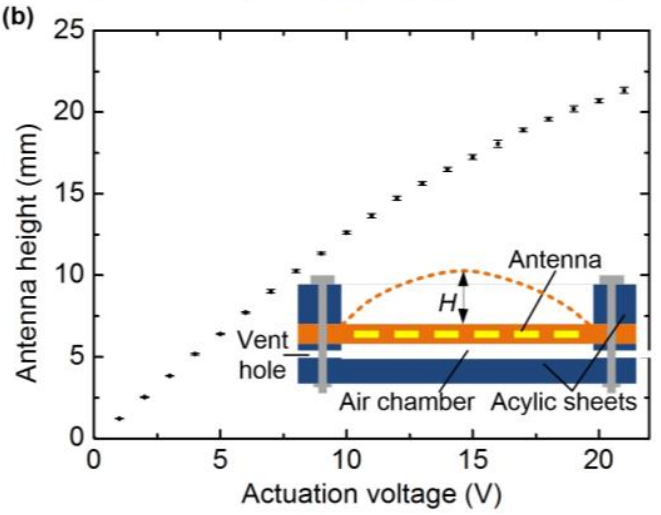

Figure 2: (a) The air pumping setup via four MEMS voltage actuated micro-blowers (b) Relationship between the inflation height and micro-blower actuation voltage with error bars.

\section{OPERATION AND MEASUREMENT}

In order to inflate the liquid metal antenna to a dome shape and well control its deformation, an inexpensive and accurate control unit is built with MEMS microblowers (MZB1001E00; Murata, Kyoto, Japan). Briefly, the microblower utilizes a piezoelectric based drive method that features small dimensions and is suitable as a source for high-pressure air generation with compact and low profile. To provide a high pressure to deform the antenna, four microblowers are connected in parallel with tubing and three-way hose fittings (Fig. 2(a)). Subsequently, the planar antenna is sandwiched between two $3 \mathrm{~mm}$ thick acrylic boards (Total Plastic, Baltimore, MD) with the help of 4 nylon screws, providing a gas tight package. The whole antenna structure is inflated by two lateral air vents pre-machined on two sides of the lower PMMA board. A circular through-hole with the diameter of $50 \mathrm{~mm}$ is created in the upper cladding board. When a D.C. voltage is applied to the microblowers, the microblowers generate high-speed airflow to pneumatically push the elastomer into a dome-like cap. The relationship between the applied actuation D.C. voltage to the microblowers and the resulting height $H$ is investigated. As shown in Fig. 2(b), the height increases almost linearly with an applied voltage at low voltage regions $(0-10 \mathrm{~V})$ and then increases slightly slowly at high voltage regions (11-21 V). The reliability of the antenna was investigated by repeatedly balloon it and deflate it from $0 \mathrm{~V}$ to $21 \mathrm{~V}$. After 100 times of ballooning, the antenna was shaped nearly the same cap height with standard deviation less than $1.5 \%$. At the maximum allowed voltage of $21 \mathrm{~V}$ for the microblowers, the obtained dome height is $21.8 \mathrm{~mm}$. During the reliability test, the liquid metal inside the channels is able to reshape and respond to the applied pressure and adapt to different cap shapes continuously every time, thus ensure the whole reconfigurable antenna remain functional without fatigue or cracking after repeated measurements.
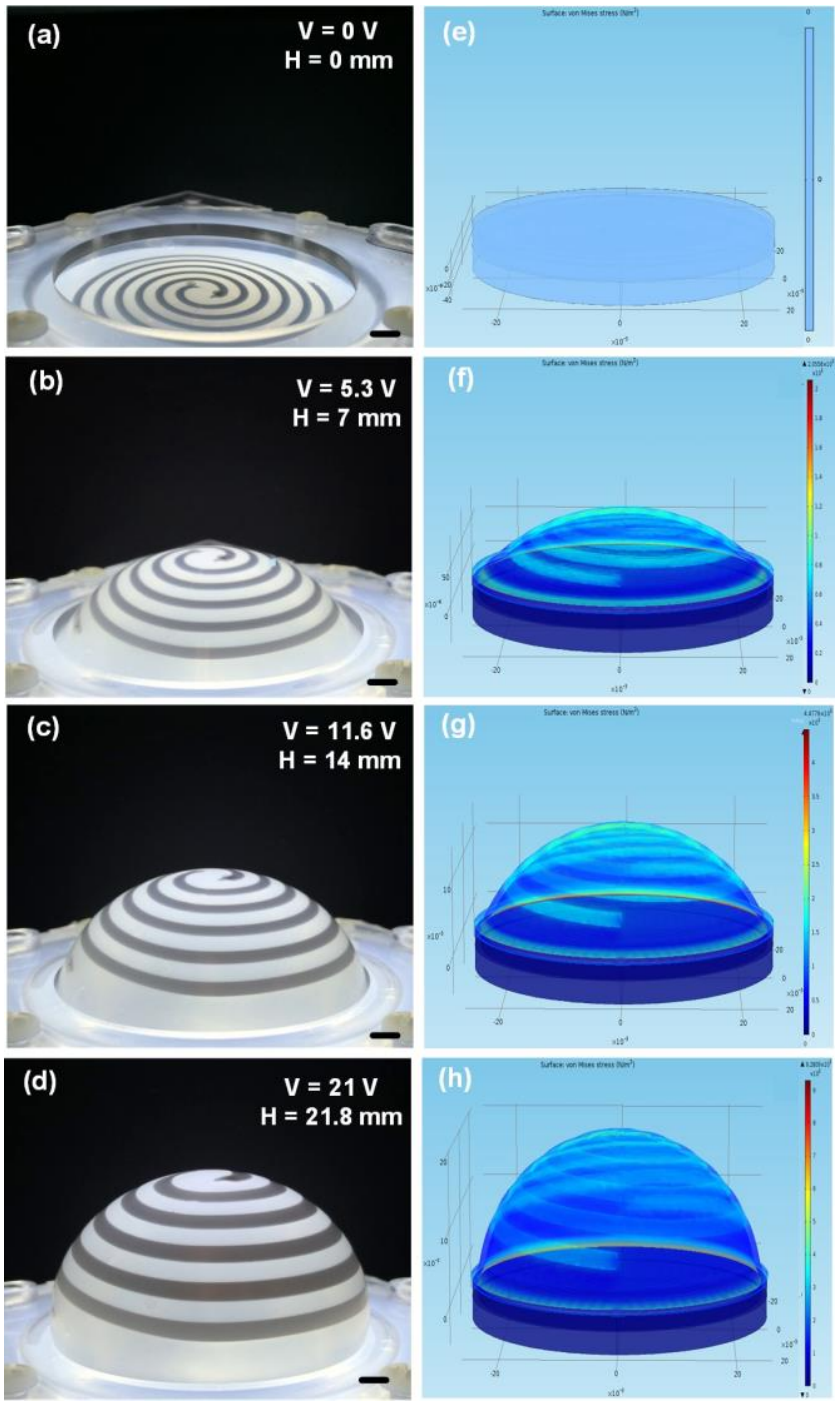

Figure 3: Time-lapse images (a-d) and FEA simulation results (e-h) for the Ecoflex elastomer containing the liquid metal antenna with different inflation heights: $0(a, e), 7 \mathrm{~mm}(b, f), 14 \mathrm{~mm}(c, g)$, and $21.8 \mathrm{~mm}(d, h)$. Scale bars in $(a-d)$ represent $3 \mathrm{~mm}$.

Fig. 3 shows the FEA simulation result of stress distribution for four instances of the antenna under different actuation voltages applied to the microblowers. The Young's modulus and Poisson's ratio of Ecoflex are $29.5 \mathrm{kPa}$ and 0.43 , respectively. The pressure data for the simulation are obtained by using a pressure sensor (SPD015AA; Smartec, The Netherlands). As the Ecoflex membrane is inflated, mechanical restoring force of the elastomer induced by the stress will eventually balance with the air pressure and achieve an equilibrium shape at a certain height of the dome. For $H=0,7$ $\mathrm{mm}, 14 \mathrm{~mm}$, and $21 \mathrm{~mm}$ under the applied pressure of $0,2.2 \mathrm{kPa}$, 
$5.2 \mathrm{kPa}$, and $11.8 \mathrm{kPa}$, the resulting maximum surface stress in the deformed Ecoflex membrane is found to be $0,184 \mathrm{kN} / \mathrm{m}^{2}, 491$ $\mathrm{kN} / \mathrm{m}^{2}$, and $915 \mathrm{kN} / \mathrm{m}^{2}$, respectively.

The electromagnetic measurement is carried out in an anechoic chamber. The antenna is fed by connecting two pins of a 3-mm SMA connector with the two heads of the spiral arms at the center region of the antenna. A programmable vector network analyzer (VNA, Agilent E8364) is used in this experiment. The radiation pattern is measured by putting the antenna towards to a horn antenna within the far field region using an automatic antenna measurement system (Dimond Engineering, CA). Fig. 4 shows the measurement setup where the antenna rotates around $z$-axis, and the dashed line is in $x-y$ plane. The rotation angle $\varphi$ is defined as the angle between the horn antenna (dashed line) and the $x$-axis. The turntable is set to rotate from $0^{\circ}$ to $180^{\circ}$ clockwise with an angular resolution of $2^{\circ} .21$ measurements are conducted at each position to minimize the effect of possible mechanical vibration.

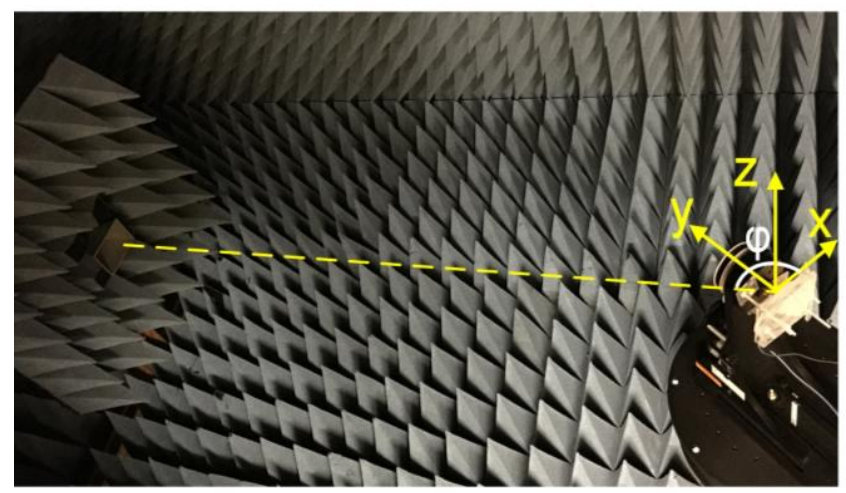

Figure 4: Photo of a setup for measuring radiation patterns of the liquid metal antenna with horizontal polarization.

\section{RESULTS AND DISCUSSION}

We measured reflection coefficient of the antenna as a function of dome heights (Fig. 5). The result shows that the passing band for this antenna spans from $6.9 \mathrm{GHz}$ to $13.16 \mathrm{GHz}$ with $\mathrm{S} 11$ lower than $-10 \mathrm{~dB}$. When the antenna is inflated, the frequency band remains in the same wide frequency region, ensuring that the antenna is fully functional in this range.

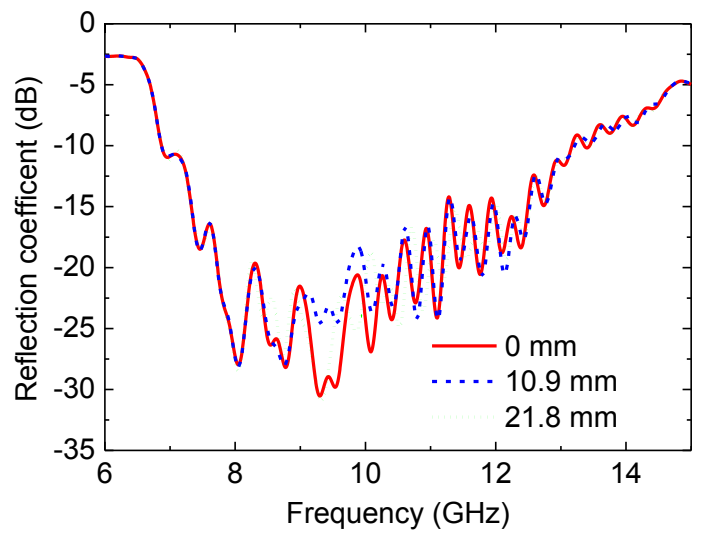

Figure 5: Measured reflected power with different inflation heights of the liquid metal antenna.

Fig. 6 shows the radiation patterns of the antenna with horizontal polarization at $8.5 \mathrm{GHz}$ for three different inflation heights: $0 \mathrm{~mm}$, $10.9 \mathrm{~mm}$, and $21.8 \mathrm{~mm}$. The result shows that the signal strength exhibits the strongest along the $y$ axis, which plotted at $90^{\circ}$ for $y$ direction and at $270^{\circ}$ for $-y$ direction. The planar antenna transmits almost the same signal strength for both $y$ and $-y$ directions, due to the geometrical symmetry at $x-z$ plane along $y$-axis. As the inflation height increases, the dome shaped antenna tends to increase the radiation strength along $y$ direction $\left(90^{\circ}\right)$, while decreasing the radiation strength along $-y$-direction $\left(270^{\circ}\right)$. Further increasing the inflation height will further enhance the radiation along $y$-direction. Therefore, the directivity at the main lobe direction ( $y$-direction) can be optimized by inflating the antenna to higher heights.

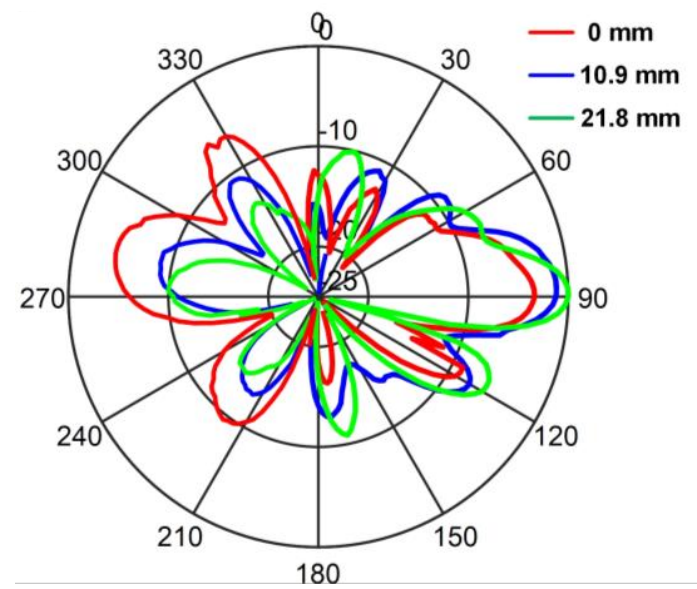

Figure 6: Measured radiation pattern at $8.5 \mathrm{GHz}$ of horizontal polarization for three inflation heights.

The radiation patterns of the antenna at different frequencies are measured in the frequency range from $7.6 \mathrm{GHz}$ to $9.8 \mathrm{GHz}$ with the reflection power lower than $-20 \mathrm{~dB}$ to ensure the antenna operate with its best performance. Fig. 7 shows the normalized radiation patterns of the antenna for three different inflation heights $(0,10.9$ $\mathrm{mm}$, and $21.8 \mathrm{~mm}$ ) at two sample frequencies $(8.5 \mathrm{GHz}$ and 9.5 $\mathrm{GHz}$ ). By rotating the receiving horn antenna by $90^{\circ}$, the radiation patterns of vertical polarization are also measured. Based on the radiation patterns shown in Fig. 7, the axial ratio (AR) of the antenna at different dome heights and operation frequencies is obtained and given in Table 1.

Table 1: axial ratio for different inflation heights and frequencies.

\begin{tabular}{c|c|c|c}
\hline \hline & $0(\mathrm{~mm})$ & $10.9(\mathrm{~mm})$ & $21.8(\mathrm{~mm})$ \\
\hline $8.5 \mathrm{GHz}$ & 1.25 & 1.54 & 1.32 \\
\hline $9.5 \mathrm{GHz}$ & 1.58 & 1.10 & 1.08 \\
\hline \hline
\end{tabular}

As discussed above, the radiation strength or directivity of the antenna can be improved only in the inflation direction of the dome. Therefore, the radiation patterns of the antenna are measured only from $0^{\circ}$ to $180^{\circ}$ along the main lobe direction or the inflation direction for meaningful use purpose. The measured radiation patterns show good directivity for all the measured heights at the two sample frequencies along the inflation direction. More importantly, as the inflation height increases, the antenna exhibits stronger directivity along its main lobe direction, thus optimizing its directivity along the inflation direction. Specifically, at $8.5 \mathrm{GHz}$, by inflating the antenna from $0 \mathrm{~mm}$ to $10.9 \mathrm{~mm}$ and further to $21.8 \mathrm{~mm}$, the signal strength is improved by $2 \mathrm{~dB}$ and further to $3 \mathrm{~dB}$ in the horizontal polarization direction, and by $1 \mathrm{~dB}$ and further to $3 \mathrm{~dB}$ in the vertical polarization direction. Similarly, at $9.5 \mathrm{GHz}$, the signal 
strength in the inflation direction is also enhanced by $3 \mathrm{~dB}$ and $6 \mathrm{~dB}$, for the horizontal and vertical polarization, respectively. It should be pointed out that as the radiation pattern shows a good similarity between the vertical and horizontal polarizations, the antenna provides good circular polarization of an electromagnetic wave. (a)

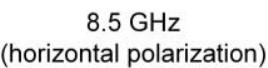
8

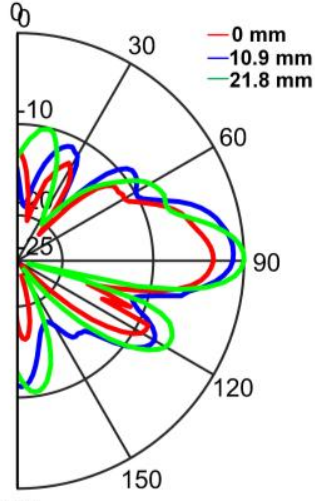

(c) 180

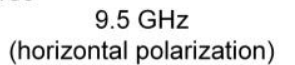

( )

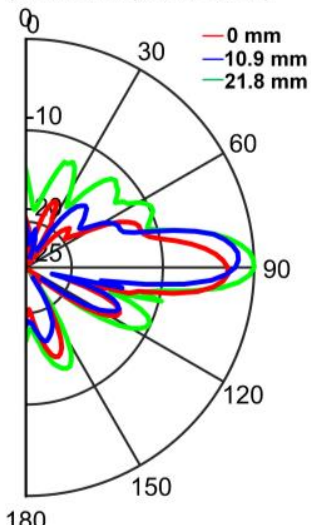

180 (b)

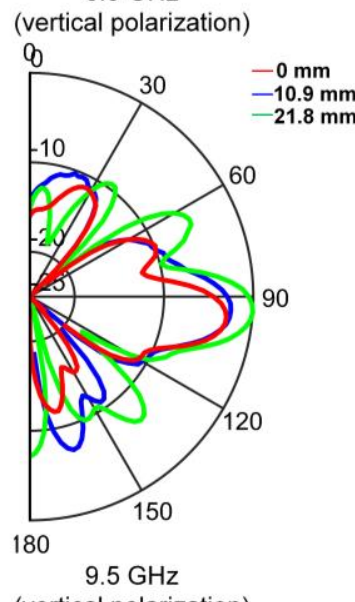

(d) (vertical polarization)

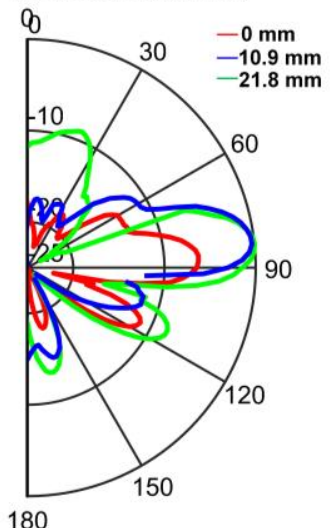

Figure 7: Measured radiation patterns of both horizontal and vertical polarizations from $0^{\circ}$ to $180^{\circ}$ for different inflation heights at two sample frequencies: $8.5 \mathrm{GHz}(a, b)$, and $9.5 \mathrm{GHz}(c, d)$.

Furthermore, benefitting from the deformability of the liquid metal and elastomer, the present antenna is able to preserve its electromagnetic properties after being inflated for 500 times to the maximum height of $21.8 \mathrm{~mm}$.

To obtain the benefit of structural tuning, the device needs to operate at temperatures that keep the infused liquid metal in a liquid state. For EGaIn used in this study has a melting point of $\sim 15.5^{\circ} \mathrm{C}$, making it liquid at room temperature. It should be pointed out that in case that lower operation temperatures are required, the device requires using other liquid metals or alloys with lower melting points. Also, theoretically, the directivity of the device can be further enhanced by adding a back cavity with a gap of a quarter of wavelength $(\lambda / 4)$ between a back reflector and the planar antenna. However, it is difficult to maintain the $\lambda / 4$ gap during the mechanical deformation of the antenna. We will optimize the device structure and introduce a cavity to the current design in the future. Nevertheless, the demonstrated reconfigurability of the present device allows for continuous tuning the radiation pattern and directivity of the antenna.

\section{CONCLUSIONS}

A directivity reconfigurable liquid metal based antenna with wide operating frequency band was developed and demonstrated. The liquid metal based antenna was fabricated by encasing the liquid metal into a highly stretchable elastomer. Benefiting from this fabrication process and structure, the antenna can withstand large deformation with great reliability and repeatability. By pneumatically inflating the antenna with MEMS micro-blower, the antenna can form spherical shapes with different heights. By increasing the inflation heights, the signal strength of present antenna can be improved to different extents from height of $0 \mathrm{~mm}$ to $21 \mathrm{~mm}$. With an improved directivity in a broad operating frequency range, the antenna can radiate more energy towards the receiver. The potential application of this antenna can be found in systems that require targeting, directivity improving and a wide operating frequency range.

\section{ACKNOWLEDGEMENTS}

This work was supported in part by the U.S. National Science Foundation under Grant \# ECCS-0954765 and the China Scholarship Council.

\section{REFERENCES}

[1] K. Hong, S. H. Kim, K. H. Lee, and C. D. Frisbie, "Printed, sub-2V ZnO Electrolyte Gated Transistors and Inverters on Plastic," Advanced Material, 25, 25 (2013).

[2] D. H. Kim, J. H. Ahn, W. M. Choi, H. S. Kim, T. H. Kim, J. Z. Song, et al., "Stretchable and foldable silicon integrated circuits," Science, 320, 5875 (2008).

[3] D. H. Kim, Z. J. Liu, Y. S. Kim, J. Wu, J. Z. Song, H. S. Kim, et al., "Optimized structural designs for stretchable silicon integrated circuits," Small, 5, 24 (2009).

[4] M. Kubo, X. Li, C. Kim, M. Hashimoto, B.J. Wiley, D. Ham, and G. M. Whitesides, "Stretchable Microfluidic Radiofrequency Antennas," Advanced Material, 22, 2749 (2010).

[5] S. Cheng, A. Rydberg, K. Hjort, and Z. Wu, "Liquid metal stretchable unbalanced loop antenna," Applied Physics Letter, 94, 144103 (2009).

[6] P. Liu, S. Yang, A. Jain, Q. Wang, H. Jiang, J. Song, T. Koschny, C.M. Soukoulis, and L. Dong, "Tunable meta-atom using liquid metal embedded in stretchable polymer", Journal of Applied Physics, 118, 014504 (2015).

[7] S. M. Yang, P. Liu, M. Yang, Q. G. Wang, J. M. Song, and L. Dong, "From Flexible and Stretchable Meta-Atom to Metamaterial: A Wearable Microwave Meta-Skin with Tunable Frequency Selective and Cloaking Effects", Scientific Report, 6, 21921 (2016)

[8] S. Cheng, A. Rydberg, K. Hjort, and Z. G. Wu, "Liquid metal stretchable unbalanced loop antenna," Applied Physics Letter, 94, 144103 (2009).

[9] M. Jobs, K. Hjort, A. Rydberg, and Z. G. Wu, "A Tunable spherical cap microfluidic electrically small antenna," Small, 9, 19 (2013).

[10] M. Wang, C. Trlica, M. R. Khan, M. D. Dickey, and J. J. Adams, "A reconfigurable liquid metal antenna driven by electrochemically controlled capillarity," Journal of Applied Physics, 117, 194901 (2015).

\section{CONTACT}

*L. Dong, tel: +1-515-294-0388; ldong@iastate.edu 